\section{Molecular characterization of acute myeloid leukemia patients who relapse more than 3 years after diagnosis: an exome sequencing study of 31 patients}

Acute myeloid leukemia (AML) is the most common acute leukemia in adults, with affected patients having a median age of 65-70 years at diagnosis. Standard chemotherapy protocols result in remission in $\sim 40-80 \%$ of patients. Relapse of the disease, however, is common and associated with a dismal outcome. Higher incidences of relapse have been observed in patients with FLT3 internal tandem duplication (ITD), TP53 alterations and adverse-risk cytogenetics. ${ }^{1,2}$ AML is known to be a genetically heterogeneous disease, ${ }^{3,4}$ a fact which also plays an important role in resistance to chemotherapy and relapse. ${ }^{5}$ Therefore, clonal evolution from diagnosis to relapse has been extensively studied in AML in order to identify mechanisms of therapy resistance. ${ }^{6,7}$ In the majority of patients, genetic changes between the two disease states can be observed and the stability of a gene mutation was found to depend on the molecular function. NPM1 mutations, one of the most common alterations in AML, were found to be relatively stable, ${ }^{8}$ similar to mutations affecting epigenetic modifiers (DNMT3A, IDH1/2 and TET2). ${ }^{7}$ By contrast, mutations that alter components of signaling pathways or transcription factors have been shown to be less stable during disease progression. ${ }^{8}$ However, gene mutations specifically driving AML relapse remain uncertain as previous studies did not identify a general relapse mechanism. The majority of relaps- es occur within the first 2 years after diagnosis and it has been suggested that patients who remain in remission for $>3$ years can be considered as cured. ${ }^{9}$ Nonetheless, late relapse of AML can occur, ${ }^{10,11}$ although comprehensive genetic data on a larger cohort of AML patients with late relapses are scarce.

Thus, we selected a total of 31 AML cases sent to our laboratory for diagnostic work-up from 2005-2015 who relapsed $>3$ years after initial diagnosis to delineate the genetic profile and gain knowledge about the molecular pathogenesis in these patients. All patients were treated intensively with chemotherapy and went into remission. Moreover, four patients received an allogeneic stem cell transplant. Based on sample availability, whole exome sequencing was carried out for 31 patients at the time points of diagnosis and relapse as well as on matched remission samples that were available for 19 patients. Remission samples after stem cell transplantation were available for all transplanted patients and also sequenced. NPM1 mutations and FLT3-ITD were identified by routine diagnostics as exome sequencing is not reliable for the detection of these types of aberrations. As a control cohort, we identified patients who relapsed within 1 year $(n=371)$ after diagnosis for comparison of cytogenetic and molecular risk factors. For exome sequencing and variant calling, enrichment based library preparation was performed using the TruSeq DNA Library Preparation Kit (Illumina, San Diego, CA, USA) and the xGen Exome Research Panel (IDT, Integrated DNA Technologies, Coralville, IA, USA) and sequenced (2x151 bp) on a NovaSeq instrument (Illumina). Further data processing, stringent filtering and the patients' characteristics are

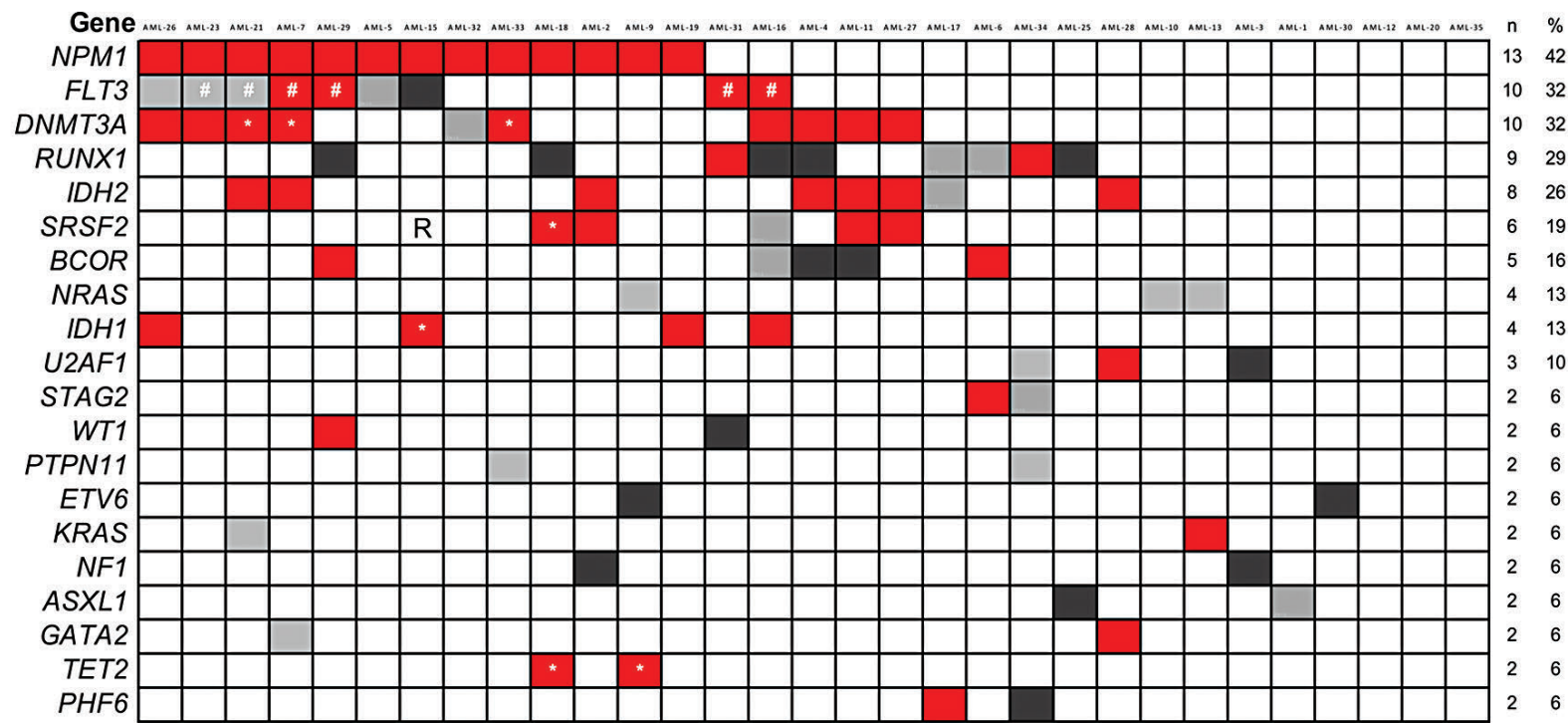

$\begin{array}{r}\begin{array}{r}\text { Time to relapse } \\ \text { Karyotype }\end{array} \\ \hline \text { NA }\end{array}$

Mutation:

$\square$ Lost at relapse $\square$ Gained at relapse $\square$ Diagnosis and relapse \# FLT3-ITD * Present at remission

Time to relapse: Karyotype:

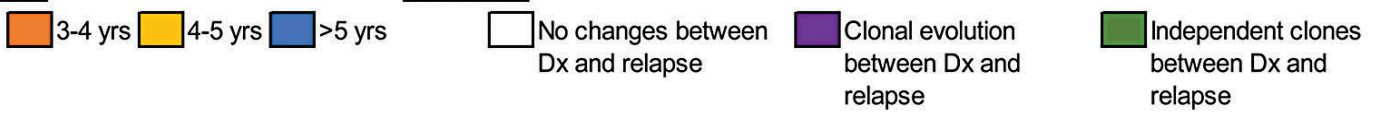

Figure 1. Mutation landscape of acute myeloid leukemia patients who relapsed more than 3 years after diagnosis. Each column represents one analyzed patient, each line one analyzed gene. NA: not available; R: detected in the remission sample only. 


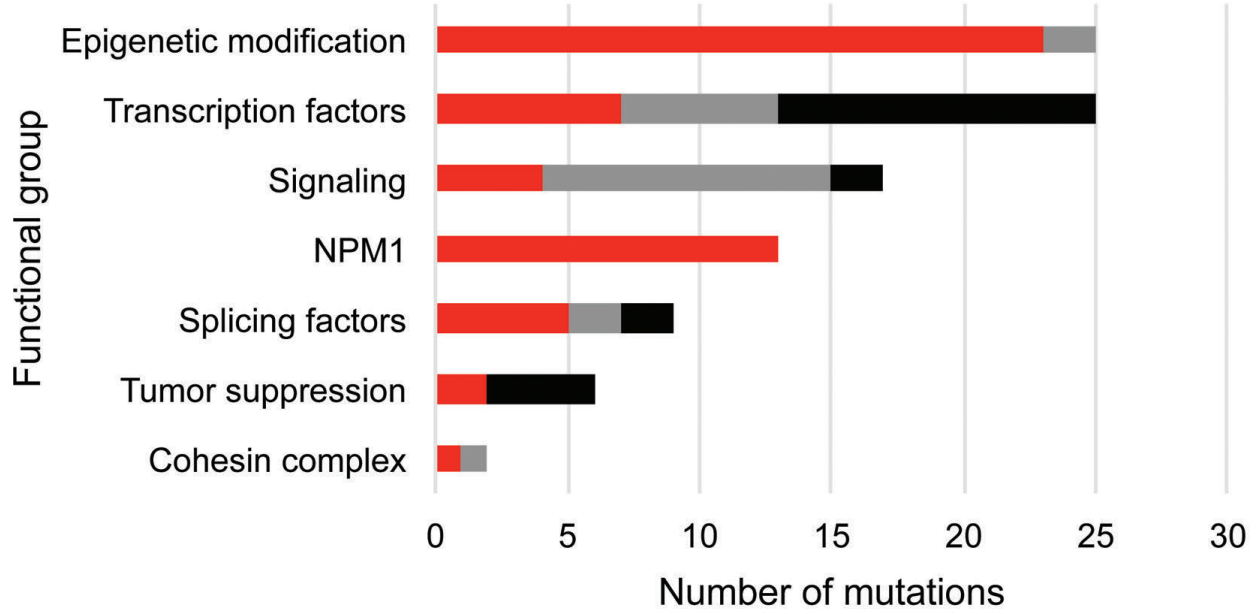

Dx and Relapse $\quad$ Lost at relapse Gained at relapse

Figure 2. Mutation frequency based on functional groups proposed to be relevant in acute myeloid leukemogenesis. Stability of the mutations between diag nostic and matched relapse samples depended on the affected gene. While mutations in epigenetic modifiers were mostly present at both time points (indicated in red), mutations in transcription factors and tumor suppressor genes were often gained at relapse (dark gray), while mutations affecting signaling pathways were often lost during disease progression (light gray);

described in the Online Supplementary Material, Online Supplementary Figure S1 and Online Supplementary Tables S1 and $S 2$.

Exome sequencing was performed on diagnostic (mean coverage: $127 \mathrm{x}$ ) and relapse (mean coverage: $115 \mathrm{x}$ ) samples. Combining data at diagnosis and relapse, we identified a total of 590 mutations (single nucleotide variants, $\mathrm{n}=524$; insertions-deletions, $\mathrm{n}=66$ ), affecting 487 different genes (Online Supplementary Table S3). Of these genes, 52 were recurrently mutated. Highest mutation frequencies were observed in DNMT3A (10/31 patients, $32 \%)$, RUNX1 (9/31 patients, 29\%), IDH2 (8/31 patients, 26\%), SRSF2 (6/31 of patients, 19\%), BCOR (5/31 patients, $16 \%$ ), and CDC42EP1, FLT3, IDH1, USF3, and VCX3B (4/31 patients, 13\%). Overall, we identified 4-34 (median: 19) mutations per patient. In more detail, we detected a median of 13 mutations (range, 4-25) at diagnosis and a median of 16 (range, 1-29) at relapse. In 30/31 patients, we identified mutations that were present at both time points, highlighting the genetic relationship between both disease states (Figure 1). However, clonal evolution by means of gained or lost mutations at relapse was noticed in every patient: 1-22 changes in the mutational pattern were observed per patient (median: 8), 0-19 (median: 3) mutations per patient were lost at relapse and 0-15 (median: 4) mutations were gained at relapse.

When comparing the matched diagnostic and relapse samples for all 31 patients, of the 590 mutations identified, 306 mutations were present at both disease time points, while 114 mutations were detected only in the diagnostic specimen and 170 mutations were exclusively identified in the relapse sample. We queried the molecular function (8 distinct groups) of the genes affected using the PANTHER gene list analysis online tool (http://www.pantherdb.org/). The frequency of the affected function was compared between all mutated genes and genes mutated either exclusively at diagnosis or relapse. This comparison of mutations revealed a slight increase in mutations affecting transcription regulation ( $8 \% v s$. $11 \% ; P=$ not significant) and decrease of mutations affecting signal transduction $(10 \%$ vs. $3 \%, P=$ not significant) towards disease progression (Online Supplementary Figure S2). Next, we limited our analyses to genes known to be recurrently mutated in myeloid neoplasms and to molecular functions relevant to the pathogenesis of AML. ${ }^{12}$ Overall, 11/22 (50\%) of mutations lost at relapse affected signaling pathways, followed by transcription factors $(n=6)$, splicing factors and epigenetic modifiers $(n=2)$ (Figure 2). In contrast, 20 mutations were gained during disease progression: $12 / 20(60 \%)$ affected transcription factors with known roles in hematopoiesis ( $R U N X 1, \mathrm{n}=6$; ETV6 and BCOR, $\mathrm{n}=2$; WT1 and BCORL1, $\mathrm{n}=1)$. The majority of these mutations are predicted to result in truncation of the coding protein. In addition, four mutations acquired in the relapse clone affected tumor suppressor genes (NF1, $\mathrm{n}=2$; TP53, $\mathrm{n}=1$, PHF6, $\mathrm{n}=1$ ). Four patients (AML-4, AML-13, AML-28 and AML-30) received an allogeneic stem cell transplant before relapse. In these patients, of the 15 mutations gained at relapse, six affected transcription (co)factors (DEPDC1, PLAG1, SPI1, ETV6, RUNX1 and BCOR) and three affected proteins involved in cell cycle regulation or apoptotic processes (PPP1R10, G2E3 and MAPK4). However, we did not observe mutations that can be linked to immuneescape mechanisms.

Data from conventional cytogenetic analyses were available for 29 patients at the time point of diagnosis and 29 patients at the time point of relapse, comparison of the disease states was possible for 27 patients. Cytogenetic changes between diagnosis and relapse were observed in 13/27 (48\%) patients analyzed at both time points. Evolution from a normal to aberrant karyotype was evident in $7 / 13$ of these patients. A complex karyotype different from the one detected initially at diagnosis was observed in $2 / 13(15 \%)$ relapsed patients and in one patient with a complex karyotype at diagnosis, further additional aberrations were detected at relapse (Online Supplementary Table S4). Furthermore, we identified emergence of cytogenetically independent clones between diagnosis and relapse in $3 / 13$ (23\%) cases.

We also performed exome sequencing of remission samples which were available for 19 patients (mean cov- 
erage: $118 \mathrm{x}$ ). In order to avoid confounding by private germline variants, we filtered for genes known to be recurrently affected in AML patients and identified a total of nine persisting mutations (DNMT3A, $\mathrm{n}=3$; TET2, $\mathrm{n}=3$; $S R S F 2, \mathrm{n}=2$ and IDH1, $\mathrm{n}=1)$ in $6 / 19(32 \%)$ patients evaluated in remission. Importantly, the remission samples were negative for all other mutations identified at diagnosis, ruling out refractory AML, but rather suggesting the persistence of a preleukemic clone. Age at diagnosis of patients with or without persisting mutations was comparable (median: 52.3 vs. 57.3 years; $P=0.8$ ). Moreover, time from diagnosis to relapse did not differ between the two groups of patients (median: 47 vs. 47 months; $P=0.8$ ). An analysis of mutations occurring after chemotherapy is described in the Online Supplementary Data and Online Supplementary Figure S3. Furthermore, four case reports are presented in the Online Supplementary Data to further explain the clonal evolution of molecular and cytogenetic alterations in AML patients with late relapses (Online Supplementary Figure S4).

We were able to show that mutations in epigenetic modifiers were mostly stable between diagnosis and relapse, suggesting that these mutations are early events in leukemogenesis. By contrast, mutations affecting signaling pathways were frequently lost while the majority of mutations in transcription factors and tumor suppressor genes were gained in the relapse clone of our patients. Prognostic markers have been established to identify AML patients with dismal outcome. ${ }^{3}$ We compared the frequency of some of these markers in our cohort of patients with late relapses to the frequency in patients who relapsed within the first year after diagnosis to check for a relationship between high-risk markers and time to relapse. Although we observed a trend towards an enrichment of high-risk parameters in patients with early relapse, this trend was not statistically significant. Similarly, in a different study no significant differences in age, AML status, white blood cell count, karyotype, FLT3-ITD mutation, CEBPA mutation and induction regimen were observed between patients with early or late relapse ${ }^{13}$ indicating that the dynamics of relapse might be influenced by other factors. In summary, we describe the clonal evolution in a large cohort of AML patients who experienced a late relapse of their disease. Our data indicate that the relapse clone is characterized by gain of mutations that mainly affect transcription factors and tumor suppressor genes, suggesting an important role in the pathogenesis. This knowledge might help to identify tailored therapy approaches to reduce the risk of AML relapse.

Luise Hartmann, Claudia Haferlach, Manja Meggendorfer, Niroshan Nadarajah, Wolfgang Kern, Torsten Haferlach and Anna Stengel

\section{MLL Munich Leukemia Laboratory, Munich, Germany}

Acknowledgments: the authors would like to thank all co-workers at the MLL Munich Leukemia Laboratory for approaching together many aspects in the field of leukemia diagnostics and research with their dedicated work. The authors would also like to thank all physicians for providing samples and caring for patients as well as collecting data.

Correspondence: ANNA STENGEL - anna.stengel@mll.com doi:10.3324/haematol.2019.227702

Information on authorship, contributions, and financial \& other disclosures was provided by the authors and is available with the online version of this article at www. haematologica.org.

\section{References}

1. Döhner $\mathrm{H}$, Estey E, Grimwade D, et al. Diagnosis and management of AML in adults: 2017 ELN recommendations from an international expert panel. Blood. 2017;129(4):424-447.

2. Rücker FG, Schlenk RF, Bullinger L, et al. TP53 alterations in acute myeloid leukemia with complex karyotype correlate with specific copy number alterations, monosomal karyotype, and dismal outcome. Blood. 2012;119(9):2114-2121.

3. Papaemmanuil E, Döhner H, Campbell PJ. Genomic classification in acute myeloid leukemia. N Engl J Med. 2016;375(9):900-901.

4. Grimwade D, Ivey A, Huntly BJ. Molecular landscape of acute myeloid leukemia in younger adults and its clinical relevance. Blood. 2016;127(1):29-41.

5. Landau DA, Carter SL, Getz G, Wu CJ. Clonal evolution in hematological malignancies and therapeutic implications. Leukemia. 2014; 28(1):34-43

6. Li S, Garrett-Bakelman FE, Chung SS, et al. Distinct evolution and dynamics of epigenetic and genetic heterogeneity in acute myeloid leukemia. Nat Med. 2016;22(7):792-799.

7. Greif PA, Hartmann L, Vosberg S, et al. Evolution of cytogenetically normal acute myeloid leukemia during therapy and relapse: an exome aequencing study of 50 patients. Clin Cancer Res. 2018;2 4(7):1716-1726.

8. Krönke J, Bullinger L, Teleanu V, et al. Clonal evolution in relapsed NPM1-mutated acute myeloid leukemia. Blood. 2013;122(1):100108.

9. Yanada M, Garcia-Manero G, Borthakur G, Ravandi F, Kantarjian H, Estey E. Potential cure of acute myeloid leukemia: analysis of 1069 consecutive patients in first complete remission. Cancer. 2007;110(12):2756-2760.

10. Verma D, Kantarjian H, Faderl S, et al. Late relapses in acute myeloid leukemia: analysis of characteristics and outcome. Leuk Lymphoma. 2010;51(5):778-782

11. Watts JM, Wang XV, Litzow MR, et al. Younger adults with acute myeloid leukemia in remission for $\geq 3$ years have a high likelihood of cure: the ECOG experience in over 1200 patients. Leuk Res. 2014; 38(8):901-906.

12. Döhner H, Weisdorf DJ, Bloomfield CD. Acute myeloid leukemia. N Engl J Med. 2015;373(12):1136-1152.

13. Bertoli S, Tavitian S, Berard E, et al. More than $10 \%$ of NPM1-mutated AML relapses occur after 5 years from complete remission. Blood. 2018;132(Suppl 1):2802. 